\title{
Corela
}

Cognition, représentation, langage

HS-12 | 2012

Langue, espace, cognition

\section{Du nom place aux verbes déplacer et replacer: quelques questions de legs et d'appropriations sémantiques}

\section{Francine Gerhard-Krait}

\section{(2) OpenEdition}

Journals

Édition électronique

URL : https://journals.openedition.org/corela/2790

DOI : 10.4000/corela.2790

ISSN : 1638-573X

Éditeur

Cercle linguistique du Centre et de l'Ouest - CerLICO

Référence électronique

Francine Gerhard-Krait, « Du nom place aux verbes déplacer et replacer : quelques questions de legs et d'appropriations sémantiques », Corela [En ligne], HS-12 | 2012, mis en ligne le 19 décembre 2012, consulté le 21 septembre 2021. URL : http://journals.openedition.org/corela/2790 ; DOI : https:// doi.org/10.4000/corela.2790

Ce document a été généré automatiquement le 21 septembre 2021.

\section{(c) (i) (2)(2)}

Corela - cognition, représentation, langage est mis à disposition selon les termes de la licence Creative Commons Attribution - Pas d'Utilisation Commerciale - Partage dans les Mêmes Conditions 4.0 International. 


\title{
Du nom place aux verbes déplacer et replacer : quelques questions de legs et d'appropriations sémantiques
}

\author{
Francine Gerhard-Krait
}

1 L'étude des verbes déplacer et replacer s'inscrit dans un travail sur la filiation morphosémantique du nom d'espace place $e^{1}$ dans lequel il s'agit de dégager les propriétés sémantiques que ce nom lègue aux différents membres de sa famille constructionnelle et la manière dont ces derniers en disposent. La problématique du fonctionnement sémantique de ces deux verbes combine des questions relevant de la sémantique des mots construits, de la sémantique de l'espace, et de celle plus spécifique des verbes de déplacement. Elle relève aussi de façon cruciale de la dimension générale du sens du nom place et des manifestations de cette généralité.

Dans leurs emplois spatiaux concrets non lexicalisés ${ }^{2}$, les deux verbes replacer et déplacer correspondent lexicalement à deux types de déplacement et illustrent deux modes d'exploitation différents du sens du nom place. Le premier est un verbe de déplacement qui utilise et respecte au plus près les spécificités sémantiques du nom en question, notamment sa vocation à la localisation substantielle ${ }^{3}$. En effet, replacer $X$ désigne un procès à polarité finale qui consiste globalement à faire changer une substance $\mathrm{X}$ de place. Pour le second, le verbe déplacer, c'est une tout autre histoire. Si le changement de localisation concerne bien des substances, et si la glose changer $X$ de place peut lui convenir dans certains cas, déplacer n'est, en revanche, nullement restreint à un changement de localisation de type place, il peut tout à fait s'agir d'un changement de lieu ou d'emplacement. Déplacer n'est pas davantage restreint à une polarité particulière, celle-ci peut-être finale, initiale, médiane ou multipolaire. Finalement, on le montrera, dans ses différents emplois, déplacer exploite et combine les informations de son préfixe dé- et de sa base place avec une grande liberté d'accommodation. Il en résulte un potentiel sémantique et syntaxique tout à fait particulier, notamment, une indétermination locative intrinsèque et une stature sémantique de superordonné. 
La mise en perspective de ces deux verbes conduit, dans un premier temps, à décrire leurs propriétés spatiales lexicales et les emplois auxquels ils donnent lieu, et dans un second temps, à montrer à partir des études menées récemment sur le N place (Huyghe 2009, Aurnague 2010) et des profils sémantiques des préfixes re- et dé-, comment ces deux lexèmes construits tirent parti, chacun à sa manière, du nom place qui figure dans leur structure.

\section{Caractérisation sémantique des verbes replacer et déplacer}

4 Ainsi qu'en témoigne l'abondante littérature sur le sujet, la description sémantique des verbes locatifs est une affaire complexe (voir par exemple Kopecka 2009), où il s'agit de déterminer les propriétés spatiales intrinsèques de ce type de verbes (cf. Aurnague 2008; Vandeloise 1986), entre autres, leur polarité aspectuelle (Boons 1985, 1987) ou locative (Sarda 2000)4. Cette entreprise revient plus précisément à déterminer l'orientation initiale, médiane ou finale, et le cas échéant les orientations multiples que les verbes décrivent. Il est nécessaire alors de distinguer ce qui relève du sémantisme verbal de ce qui relève de la variation aspectuelle en discours pour laquelle de nombreux paramètres interviennent ${ }^{5}$.

5 Les verbes déplacer et replacer sont des verbes dits causatifs de déplacement (Borillo 1998, 119) parce qu'ils engagent un sujet, agent ou cause, et une cible qui, pour ces deux verbes, se réalise soit sous la forme d'un SN complément d'objet direct (Lili déplace/replace sa chaise), soit sous la forme pronominale se (Lili se déplace de quelques mètres, Lili se replace sur scène), selon en gros que la cible se meut par elle-même ou non. On exclura de l'étude les emplois pronominaux du type de (1) où il s'agit d'une lecture médio-passive (cf. ZribiHertz 2008) dans laquelle le verbe décrit la propriété de "déplaçabilité » ou de « replaçabilité » d'une entité cible dénotée par le sujet grammatical dans un rôle de patient :

(1) Ce meuble se déplace facilement / cette pièce se replace facilement ${ }^{6}$.

Dans la mesure où l'alternative de la réalisation du cod en SN dét. + Nom / Pronom est conditionnée par le trait de mobilité intrinsèque de la cible, nous considérons qu'il n'y a pas lieu de traiter différemment les deux types de structure illustrées par les exemples $(2-4)$ :

(2) Pierre se déplace à pied / Pierre déplace les meubles à l'aide d'un diable.

(3) Pierre se déplaça de quelques mètres / Pierre déplaça un meuble de quelques mètres.

(4) Pierre se replaça près de la cheminée / Pierre replaça le livre sur l'étagère.

\subsection{Caractérisation sémantique du verbe replacer}

7 Le verbe replacer accepte la glose générale « changer X (la cible) de place, mettre $\mathrm{X}$ à une autre place », laquelle convient parfaitement à l'énoncé (5) :

(5) Amélie se leva, prit à quelques pas d'elle un chevalet pour le replacer à une assez grande distance du noble groupe, près d'une cloison grossière qui séparait l'atelier d'un cabinet obscur [...]. (Honoré de Balzac, 1842, La Vendetta), 
qui décrit un procès au début duquel le chevalet (la cible) occupe une certaine place, et au terme duquel il occupe une autre place, laquelle se situe à une assez grande distance du noble groupe, près d'une cloison grossière qui séparait l'atelier d'un cabinet obscur.

9 Toutefois, dans de nombreux cas, sans doute la majorité, la glose la plus adéquate est «mettre X à la place qu'il occupait précédemment». Cette variable n'a pas de répercussion particulière sur la caractérisation aspectuelle ou locative du verbe replacer. En effet, la différence entre ces deux gloses, tout comme la prédominance de la seconde, relève du fonctionnement de la préfixation en $r^{-{ }^{7}}$, et plus précisément de sa tendance à construire des procès qui, comme celui de l'énoncé (6), réitèrent une situation antérieure, en l'occurrence, une localisation antérieure de la cible:

(6) On raconte que Charles Lamb aimait tant ses livres, qu'ayant achevé une lecture, il effleurait toujours le volume de ses lèvres avant de le replacer sur son rayon. (Julien Green, 1998, 2006, Le grand large du soir, Journal : 1977-1998).

\subsubsection{Les localisations initiale et finale}

10 Le verbe replacer construit des procès à deux localisations spatiales, l'une initiale qui est à modifier et l'autre finale. En discours, lorsque replacer décrit l'itération d'une localisation antérieure, on dispose généralement d'informations explicites ou implicites sur ces deux localisations, et ces informations sont accessibles selon diverses procédures. La localisation finale, par exemple, est spécifiée par un SN locatif à déterminant défini possessif son rayon en (6), elle est induite par une relation de localisation quasi partitive entre dentier et bouche en (7), elle est accessible en (8) via nos connaissances du monde, et en particulier par ce qu'induit une bonne pratique du ménage, qui consiste à déplacer les meubles avant de balayer puis à les remettre en place après le balayage. Cette localisation est indiquée par la relative locative où ils espèrent pouvoir replacer un jour le drapeau tricolore et précisée par l'antécédent du pronom relatif le haut du clocher en (9):

(7) [...] je comprends que c'est le dentier qui a jailli hors de la bouche du mari au moment de sa chute et qu'elle s'efforce à présent de replacer discrètement, en tournant le dos au public, par décence, s'abritant et l'abritant de son mieux. (André Gide, 1939, Journal : 1889-1939).

(8) Cristina, en toilette de ville, est assise au premier plan, songeuse et parait attendre. Au fond, deux garçons, qui viennent de balayer achèvent de replacer tables et sièges. (Édouard Bourdet, 1931, Le Sexe faible).

(9) [...] venus se mêler les émigrés de Strasbourg, de Metz, de Colmar et de Mulhouse, qui, tous, ont le coeur tourné vers le haut clocher où ils espèrent pouvoir replacer un jour le drapeau tricolore. (Maurice Barrès, 1907, Mes Cahiers).

11 La localisation initiale de la cible, ou si l'on préfère le site " point de départ » du procès de modification de la relation spatiale est une donnée récupérable selon des processus tout aussi variés. Si l'on considère (7), il s'agit indirectement de l'endroit où le dentier a chu après avoir jailli hors de la bouche du protagoniste.

Replacer appartient ainsi à la catégorie des verbes qui offrent plus d'une information locative et plus précisément semble-t-il à la catégorie de ce qu'Aurnague (2008) nomme les verbes à double changement de relation locative. Généralement, le double changement n'est pas neutre, l'une des deux phases est saillante et définit le type de polarité. La phase inverse représente alors, selon le cas, une tension vers l'origine ou le but. Pour replacer, la saillance est finale; la cible ne peut être replacée que lorsque le site final est atteint. La prégnance du site final est d'autant plus marquée dans les emplois pour lesquels ce site correspond à une localisation antérieure ; une localisation inscrite dans le contexte large 
ou étroit du discours. Cette localisation antérieure de la cible est justement ce qui fait l'objet de l'itération construite par le préfixe re-. Dans ces emplois, la localisation initiale de la cible du verbe replacer correspond à la localisation finale d'un procès de changement de localisation antérieur du type de déplacer. Ce procès antérieur est clairement énoncé en (10) avec la coordination des deux verbes déplacer et replacer la table, et en (11) avec elle prit le cigare :

(10) [...] ne l'avaient pas suffisamment fêtée, et qui, de surcroît, s'étaient empressés autour d'Adélaïde pour l'aider à se relever, lui trouver une place, déplacer et replacer la table une fois qu'elle se fut assise. (Hector Bianciotti, 1985, Sans la miséricorde du Christ).

(11) Puis elle prit le cigare et en tira deux bouffées avec un air exagérément extatique avant de le replacer entre les lèvres de son mari. (Robert Sabatier, 1972, Trois sucettes à la menthe).

La localisation finale de la cible de replacer correspond alors à la localisation initiale du procès antérieur selon une formule du type de :

loc (i) de replacer $=$ loc (f) de déplacer et loc (f) de replacer $=$ loc (i) de déplacer

En somme, la localisation initiale de la cible de replacer correspond à la localisation finale de la cible de déplacer et la localisation finale de la cible de replacer correspond à la localisation initiale de la cible de déplacer. Dans ce schéma, le site de localisation finale du verbe replacer est préconstitué. Dans l'énoncé (10), par exemple, on comprend bien qu'au terme du procès replacer, la table (cible) se trouve localisée à l'endroit où elle se trouvait avant d'avoir été déplacée. Dans l'énoncé (11), on comprend tout aussi bien que le personnage a pris le cigare des lèvres de son mari et, qu'au terme du procès replacer, le cigare se trouve à nouveau entre les lèvres de ce dernier.

Replacer décrit, dans ces cas, une situation complexe où interviennent explicitement ou par inférence de multiples informations locatives qui se font écho.

\subsubsection{La dimension médiane}

On vient de le voir, pour le verbe replacer, l'essentiel de l'information concerne le changement locatif avec une nette polarité vers la localisation finale et une nécessaire prise en compte de la localisation initiale; ce qui n'est pas valable, par exemple, pour le verbe placer qui ne décrit que le site de localisation final. Du point de vue aspectuel, replacer est intrinsèquement borné à droite et à gauche, il peut néanmoins s'ouvrir aux informations médianes, même si celles-ci ne parviennent jamais à ruiner la visée du terme du procès. La présence de compléments de manière de mouvement ${ }^{8}$ (sur ce sujet, voir par exemple Geuder 2009 et Stosic 2009), comme en (12), ou celle de compléments de distance (5), ne font respectivement qu'induire une certaine durée, ou repousser le terme du procès :

(12) Elle replace précautionneusement, lentement, rapidement le collier dans son écrin.

On relève bien des emplois contextuellement non bornés, mais exclusivement dans des lectures pluriactionnelles, habituelles, fréquentatives (cf. Kleiber 1987) ou d'autres lectures présentant une pluralité d'occurrences de replacer soit via les temps verbaux (13-15), soit en présence d'un SN cible au pluriel (15-16), soit en présence d'adverbe ou autres formes de complément de fréquence (15), par exemple, et souvent en présence de plusieurs de ces facteurs comme en (15-17), entre autres illustrations : 
(13) Il commençait par mettre le Hérédia dans la cuvette d'émail qui se trouvait sur une chaise, parce que toute la table était prise. Il le replaçait ensuite sur la table de nuit, où il le retrouvait le soir, ce qui l'obligeait à le relire (il n'avait pas le courage d'aller chercher autre chose). (Alexandre Vialatte, 1951, Les Fruits du Congo).

(14) [...] pour toutes ces porteuses des chemins; de solides tables en granit, à hauteur humaine, permettant de se débarrasser un moment de la charge, et de la replacer ensuite sur la tête, sans avoir la fatigue de se courber jusqu'à terre [...]. (Pierre Loti, 1903, L'Inde (sans les Anglais)).

(15) [...] dans notre maison de Saint-Hilaire et qui (estimant sans doute que parallèle ou perpendiculaire et plaisante ordonnance ne sont pas compatibles) replaçait systématiquement en position oblique les objets qu'elle avait déplacés pour essuyer le dessus d'un meuble? (Michel Leiris, 1966, La Règle du jeu : 3 : Fibrilles).

(16) Les cartes qui sont tombées de l'aiguille peuvent ne plus être en ordre, et l'on doit les replacer à la main, à moins que l'ordre n'ait pas d'importance. Le reclassement peut se faire en ouvrant le paquet à l'endroit convenable et en replaçant la carte tombée. (John-Lionel Jolley, 1968, Le Traitement des informations).

(17) Je soulevai mes valises pleines : trop lourdes. Ceux qui ne voyagent pas souvent connaissent ce déchirement : replacer où ils étaient des livres, des objets qui ne vous suivront pas. (Violette Leduc, 1964, La Bâtarde).

Replacer est donc bien un verbe à double changement de localisation dont la polarité est finale, il est ouvert sur la manière de mouvement et sur la pluralité d'occurrences, mais il ne construit jamais une unique occurrence durative. Il n'a pas de dimension médiane intrinsèque. Le préfixe re- n'a pas de responsabilité dans cette absence puisque, par ailleurs, il est capable de construire des verbes, qui comme remarcher dans cet appareil remarche par exemple, ont des emplois clairement duratifs.

\subsection{Caractérisation sémantique du verbe déplacer}

19 Si la caractérisation sémantique (locative / aspectuelle) des verbes de déplacement est une affaire délicate, elle l'est encore davantage pour le verbe déplacer. En effet, il est particulièrement difficile d'appréhender le sens de ce dernier à partir, par exemple, des gloses synonymiques que l'on peut offrir de ses différents emplois. Sur la base des synonymes proposés dans le dictionnaire numérique du Crisco, on peut évoquer le nombre et surtout la variété locative des verbes avec lesquels déplacer entre en synonymie discursive. On relève, par exemple, changer de place / de lieu', enlever, quitter, aller, se rendre, se mouvoir, et même voyager, lesquels dans leur totalité illustrent au moins trois types de polarité aspectuelle / locative.

\subsubsection{Une polarité locative lexicalement indéterminée}

20 Comme on l'a montré dans Gerhard-Krait (à par.), en discours, la variabilité locative du verbe déplacer peut s'expliquer par le fait que ce verbe présente les trois dimensions initiale, médiane et finale du déplacement, il les implique toutes mais ne pose aucune saillance a priori. Les trois phases du procès et les localisations afférentes peuvent alors être actualisées séparément ou en combinaisons comme le montrent les exemples cidessous classés par type de saillance :

21 (i) La focalisation sur la localisation finale témoigne d'un double changement de place ou de lieu à saillance finale. Dans ces emplois, déplacer entre en synonymie discursive avec un ensemble de procès à polarité finale mettre ailleurs, attribuer une autre place / une meilleure place, aller ailleurs, venir / aller à, etc. Le changement de place à proprement 
parler est illustré par les exemples (18-20) et le changement de lieu par les exemples (21-22) ci-dessous :

(18) [...] les femmes vont de bête en bête jusqu'au bout de la longue file. Dès qu'elles ont fini d'en traire une, elles la déplacent, lui donnant à pâturer un bout de verdure intacte. (G. de Maupassant, 1884, Contes et nouvelles).

(19) David et Olivier se déplacèrent pour mieux voir les deux hommes [...]. (R. Sabatier, 1985, David et Olivier).

(20) Il décida de se tenir bien droit comme les autres, mais en se redressant, il fit un faux mouvement et fit jaillir du jus de melon sur la nappe. Il déplaça son verre pour cacher la tâche. (R Sabatier, 1972, Trois sucettes à la menthe).

(21) Une nuit, en garde, le standard m'a dit qu'une femme voulait me faire déplacer au Diable Vauvert, pour que je lui prescrive des laxatifs. (M. Winckler, 1998, La maladie de Sachs).

(22) Remarquez bien, il se déplacerait sûrement, il vient bien jusqu'ici acheter ses journaux [...]. (M. Winckler, 1998, La maladie de Sachs).

(ii) On relève aussi un double changement de localisation avec une saillance vers la localisation initiale, que l'on peut associer à des synonymes tels que quitter, enlever, et qui insiste sur le fait que la cible quitte une localisation initiale ou encore qu'au terme du déplacement la cible n'est plus à la place qu'elle occupait initialement. Ce type de lecture illustré en (23-24) a semble-t-il un caractère axiologique négatif et repose sur la possibilité d'envisager un site de localisation initiale comme étant le site de localisation le plus adéquat, le plus légitime (cf. section 2.1) ; ce qui a pour conséquence qu'au terme du procès déplacer, les cibles ne sont plus à la «bonne » place, à la place qui leur était assignée ${ }^{10}$ :

(23) Il n'eût qu'à dire un mot pour que l'ouvreuse déplaçât deux spectateurs. (R.

Radiguet, 1923, Bal).

(24) On a déplacé les objets, quel bazar !

parfaitement indécidable ou indifférente dans certains cas (probablement nombreux). En effet, en (26), une glose en « quitter le domicile » qui marque une polarité locative initiale, ou "aller à l'église", laquelle marque plutôt une polarité locative finale, semble parfaitement équivalente :

(26) [...] ça y est : le sermon. Pour ça, j'ai pas à me déplacer pour écouter le sermon, j'ai tout à domicile. (P. Cauvin, 1976, Monsieur Papa).

(iii) On relève également des emplois polarisés sur la phase médiane. Cette phase correspond à ce qui est généralement décrit comme un changement d'emplacement ${ }^{11}$ (Borillo 1998 : 39-41, Aurnague 2008 et dans ce même numéro) durant lequel la cible est vue comme mobile. La phase médiane se manifeste pleinement et strictement dans les phrases génériques et/ou habituelles, pour lesquelles déplacer engage essentiellement la capacité à ou la manière de se mouvoir dans l'espace dans des lectures pluriactionnelles par défaut comme en (27), lesquelles jouent sur les occasions de se déplacer. Mais on relève aussi des lectures à une occurrence de procès non borné comme en (28)-(29) :

(27) Il ne se déplace plus / Il se déplace lentement.

(28) Les aiguilles d'une montre se déplacent de droite à gauche.

(29) Il se déplace lentement vers le nord / à $40 \mathrm{Km} / \mathrm{h}$. 
(iv) Par ailleurs, la saillance de la phase médiane n'invalide ni la possibilité de borner le procès comme en (30), par exemple, où le début et le terme sont induits par un complément de durée, ni celle d'indiquer des localisations initiale et finale comme en (31) :

(30) « Pendant cinq à six minutes, il se déplaça avec furie, insensible à la douleur ».

(J.-P. Manchette, 1976, Trois hommes à abattre).

(31) Il se déplace à pied de village en village.

(v) Inversement, lorsque déplacer s'interprète comme un changement de place ou de lieu, il est possible d'actualiser de multiples informations sur le parcours via notamment des spécifieurs relatifs à la manière du déplacement, des indications de durée ou de distance, comme le montrent les exemples (32)-(35) :

(32) Il déplaça rapidement la commode [d'un bout à l'autre de la chambre].

(33) Il déplaça difficilement / lentement sa lourde chaise [de quelques mètres].

(34) Il mit une heure pour déplacer le bahut [de quelques mètres].

(35) Tu déplaces le fauteuil en pivotant [jusqu'à moi] et t'assieds. (M. Winckler,

1998, La maladie de Sachs).

Il est également possible d'actualiser des informations sur la destination, qui apparaît alors comme le terme potentiel ou prévisible du déplacement en cours, ce que montrent les exemples (36-37) :

(36) La voiture se déplaçait à environ quarante kilomètres heures [en direction de la fermette]. (J.-P. Manchette, 1972, Nada).

(37) Un laveur de carreaux. Pendu sur son fil, araignée, funambule, il se déplace rapidement [vers le haut]. Son parcours est lisse. (J. Vautrin, 1979, Bloody Mary).

\subsubsection{Un verbe de déplacement général}

Déplacer ne peut pas se prévaloir d'une polarité particulière. Cette sous-détermination aspectuelle et locative permet de comprendre que ce verbe serve en quelque sorte de terme superordonné (faible intension, large extension) pour la classe des prédicats locatifs dynamiques. En effet, à la différence des verbes sous-ordonnés (cf., pour une partie d'entre eux, la typologie d'Aurnague 2008: 1912, et de Boons 1987: 11) qui décrivent lexicalement des fragments de déplacement, il offre tout l'éventail des combinatoires aspectuelles / locatives possibles. Son interprétation en discours sera ainsi particulièrement sensible aux spécifieurs aspectuels et locatifs co- et contextuels de tous ordres. En somme, déplacer a autant d'emplois qu'il y a de possibilités de séquençage d'un déplacement et de possibilités de combinaison de séquences. Si, comme le souligne Aurnague (2008: 1911), un déplacement au sens strict est un changement de relation locative et d'emplacement, le verbe déplacer est l'illustration parfaite de ce qu'est un déplacement complet. A ce propos, dans de nombreux emplois, comme en (38), déplacer peut être glosé par «aller d'un endroit à un autre » associant aux pôles initial et final statiques l'aspect dynamique :

(38) Il se déplace [de village en village] dans une voiture particulière dont il a spécialement aménagé l'arrière pour y disposer son stock de parfumerie. (G. Perec, 1989, Voeux).

\subsection{Récapitulatif}

Le verbe replacer est un verbe à double changement de localisation. Comme il construit un procès d'altérité locative, il décrit forcément les deux localisations et donc les sites initial 
et final avec une polarité sur la localisation finale. Du point de vue aspectuel, c'est un verbe intrinsèquement borné. On l'a montré, il accepte des spécifieurs médians mais ces derniers ne parviennent jamais à inhiber la localisation finale, et on ne relève aucun emploi strictement médian. La dimension médiane n'est pas une donnée lexicale, mais une donnée discursive, qui ouvre sur des lectures exclusivement pluriactionnelles.

Le verbe déplacer, quant à lui, présente des emplois à double changement locatif qui se laissent aussi gloser par «changer de place, mettre à une autre place » et qui semblent pouvoir rapprocher les deux verbes des points de vue locatif et aspectuel. Mais, à la différence de replacer, il n'est pas toujours possible de dire avec certitude si la saillance est finale ou initiale. Pour replacer et déplacer, la différence fondamentale tient au fait que déplacer est largement et intrinsèquement ouvert aux informations médianes, qu'il peut convoquer avec ou sans les informations initiale et finale.

\section{Incidence du N place dans le sémantisme de replacer et déplacer}

33 Le constituant nominal place ${ }^{12}$ joue bien évidemment un rôle dans le sémantisme des verbes replacer et déplacer. Une mise en relation des propriétés sémantiques du nom place et de celles des constituants préfixaux re- et dé-, nous permettra, dans cette section, d'en évaluer l'incidence.

\subsection{Les propriétés sémantiques du $\mathrm{N}$ place}

34 Comme l'a montré Huyghe (2009), le nom place dans ses emplois concrets est un nom général d'espace spécialisé dans la localisation substantielle. En effet, une place est une portion d'espace dédiée à une substance (la place de l'armoire ; c'est la place de ma grand-mère ; ${ }^{*}$ 'est la place du concert), par opposition au N lieu (le lieu où se déroule le concert; un lieu de villégiature; *le lieu de ma grand-mère), par exemple, spécialisé dans la localisation événementielle. Le nom place se caractérise par une série de propriétés inhérentes et afférentes: il a donc un contenu sémantique général vague qui permet une infinité de possibilités référentielles (trouve une place à cet objet, n'importe laquelle!), il signale qu'une entité est localisée et qu'elle est donc statique. En outre, une place a une extension spatiale relative à celle de la substance qui l'occupe, elle est mesurable, même si, comme le précise Aurnague (2010), la correspondance extensionnelle est plus ou moins précise selon le mode de spécification qui permet de les situer dans un cadre de référence.

\subsection{Incidence pour replacer}

Pour le complexe replacer, le préfixe re-joue un rôle dans le fait qu'il y a une information à dupliquer puisqu'il a globalement pour fonction de pluraliser quelque chose en rapport avec sa base. Il s'agit bien ici d'un procès qui vise à réitérer pour une entité cible une localisation du type de place. La généralité du sens de place, sa pauvreté descriptive et donc son potentiel référentiel, tout comme sa fonction de localisation substantielle et la staticité qui l'accompagne font que pour replacer les deux localisations initiale et finale peuvent clairement avoir valeur de place, même si, d'une part, les deux places en question n'ont pas toujours le même statut et si, d'autre part, la cible peut être localisée dans un 
cadre de référence qui désigne une portion d'espace bien plus vaste que celle requise par son étendue. En effet, en (39):

(39) Il replaça le trésor dans la clairière, dé cible, déplacer peut réaliser un changement de place, de lieu ou un changement d'emplacement. Les entités mobiles par elles-mêmes peuvent changer de place, de lieu ou d'emplacement. Les entités qui ne se meuvent pas toute seules peuvent changer de place et d'emplacement dans un lieu scénique (cf. Sablayrolles 1995) ou lieu de référence (cf. Laur 1991), mais plus difficilement de lieu, si l'on considère l'étrangeté de (42) :

(42) ? J’ai déplacé le meuble de Paris à Marseille. 

succession de changements de lieux à l'intérieur d'un lieu englobant dans des lectures habituelles :

(47) Sur le campus, Pierre se déplace d'un bâtiment à l'autre en trottinette.

in, le changement d'emplacement peut constituer une succession continue de positions dans l'espace, les positions en question n'ont alors pas de prégnance et seule la mobilité de la cible constitue une information saillante :

(48) Le véhicule se déplace à $40 \mathrm{~km} /$ heure.

51 On le voit, déplacer utilise essentiellement la localisation substantielle propre à place. Tous les autres traits, le formatage extensionnel, l'immobilité de la cible, l'assignation exclusive d'une portion d'espace à une cible, sont soit exploités à la lettre, soit accommodés de manière à les rendre moins contraints. En effet, le passage de place (48) à lieu (49) :

(49) Pierre a déplacé le meuble de quelques centimètres,

(50) Le médecin s'est déplacé chez sa patiente,

rend caduque la correspondance extensionnelle du site et de la cible et le trait de staticité. Le changement continu d'emplacement :

(51) Le véhicule se déplace à $40 \mathrm{~km} / \mathrm{h}$, 
annule tout bonnement la possibilité d'une
la description d'une cible en mouvement.

\subsubsection{Le rôle de dé(s)-}

54

Le préfixe dé- construit en gros des verbes négatifs généralement à polarité initiale stricte (Boons 1984, 1985, 1987, Gerhard-Krait 2000). Si l'on s'en tient aux verbes de la souscatégorie structurelle à laquelle déplacer appartient, ils décrivent ce qui peut être vu comme un déplacement d'entité (démouler un gâteau, dépoter une plante, déterrer un os, dégainer une arme), leur base peut donc représenter le site initial ${ }^{15}$, et le SN complément d'objet direct peut être conçu comme désignant l'entité cible. Dans la grande majorité des cas, ces verbes ne décrivent pas le site final, il s'agit simplement de faire «sortir » une entité de son site de localisation initial, lequel est souvent une entité englobante ou une entité contenant. Les sites et les entités cibles en question entretiennent des relations de localisation préconstruites du type de contenant à contenu ou autres relations lexicales topologiquement contraintes (partie / tout, contiguïté spatiale, etc.).

Sur ce point, avec déplacer on relève une première entorse. Comme l'avait noté Boons (1985), dans les emplois qu'il passait en revue dans son étude, ce verbe implique « deux places ». Ce constat ne l'a pas amené à remettre en cause sa polarité initiale stricte, mais à considérer qu'il décrivait une extension vers une localisation finale qui le rapprochait d'un verbe comme transvaser, à cela près que le passage de vase à vase est construit par le préfixe trans-. Le fait que déplacer, contrairement à déloger, puisse décrire deux localisations - ce que peuvent faire également le verbe délocaliser et probablement le verbe déménager ${ }^{16}$-, tient, comme pour le verbe replacer, à la généralité du sens de place et à son grand potentiel référentiel, mais aussi au fait qu'une fois le déplacement amorcé, l'entité qui a quitté sa localisation initiale ne peut plus être à la même place. Un déplacement aussi infime soit-il conduit forcément à terme à une nouvelle localisation, et éventuellement à une nouvelle place $:$ il déplaça presque imperceptiblement sa main a pour résultat que la main n'est plus à la place qu'elle occupait dans la situation initiale.

Dans le sens de dé-, déplacer puise la valeur négative et l'orientation initiale qui se traduit toujours par la négation de la localisation initiale et celle de la staticité initiale de la cible propre à la localisation du type de place. Sa présence induit donc une prise en compte de la phase et / ou de la localisation initiale. Dans l'orientation initiale stricte associée à dé-, déplacer puise aussi la possibilité de ne pas informer sur le terme du procès (la localisation finale), à l'image de ce qui se passe pour les verbes déloger, démouler, etc. Mais contrairement à ces deux verbes, lorsque déplacer ne décrit pas de localisation finale, il n'actualise que le trait initial de staticité de la cible, et l'information locative initiale (i.e. le site initial) n'a alors aucune forme d'informativité. Dans ce cas, déplacer décrit un procès médian de changement continu d'emplacement (la voiture se déplace / roule à $40 \mathrm{~km} /$ h), un procès de négation de staticité (la cible se meut), ce dont n'est pas capable le verbe délocaliser par exemple, sans doute en raison de la nature événementielle des entités amenées à être délocalisées.

Le changement de localisation qui implique deux places, au sens le plus strict du terme, apparaît alors comme le scénario le plus contraint, c'est-à-dire impliquant le plus grand nombre de traits (staticité de la cible sur le site, correspondance extensionnelle du site et de la cible, possibilité d'une correspondance normative). C'est aussi le scénario le plus attendu, le plus proche, le plus représentatif de la structure morpho-sémantique du verbe 
déplacer. Grâce à divers types d'accommodations, déplacer parvient bien à désigner isolément ou conjointement les trois dimensions d'un procès de déplacement. La sousdétermination référentielle $\mathrm{du}$ nom place semble tenir lieu de levier à celle du lexème dont il est un des constituants.

Le verbe déplacer illustre parfaitement la complexité du fonctionnement sémantique des unités construites et confirme, si cela était encore nécessaire, que le sens des mots construits obéit à des mécanismes probablement hétérogènes qui ne sont que partiellement appréhendables a priori.

\section{Conclusion}

Les différents points abordés dans cette étude mériteraient bien entendu des précisions, néanmoins on a cherché à montrer que le nom place a une incidence particulière dans le sémantisme des verbes replacer et déplacer. Pour le premier, il restreint les informations locatives aux seuls sites initial et final, lesquels se conforment aux spécificités locatives de place : il s'agit toujours de redonner une ou sa place à une entité. Le nom place détermine les informations locatives et aspectuelles pour ce verbe. Pour déplacer, les choses sont bien plus complexes. La variété de ses emplois résulte d'une grande liberté d'exploitation et d'accommodations des propriétés sémantiques de ces constituants. La valeur négative et la polarité initiale du préfixe dé-, ajoutée à la spécificité locative et à la généralité du sens de place, fournissent les points d'ancrages sémantiques du verbe construit déplacer. Mais celui-ci, de généralisation en généralisation, construit une réelle indétermination aspectuelle et locative. Déplacer est bel et bien capable de décrire tout type de déplacement.

\section{BIBLIOGRAPHIE}

Amiot, D., 2002. «Re-, préfixe aspectuel ? ", Cahiers Chronos 10, 1-20.

Amiot, D., 2008. « La catégorie de la base dans la préfixation en dé- », in B. Fradin (ed), La raison morphologique. Hommage à la mémoire de D. Corbin. Linguisticae Investigationes Supplementa 27, 1-15.

Apothéloz, D., 2005. «Re- et les différentes manifestations de l'itérativité », Pratiques 125-126, 48-71.

Aurnague, M., 1996. «Les noms de localisation interne, tentative de caractérisation sémantique à partir des données du basque et du français », Cahiers de Lexicologie 69, 159-192.

Aurnague, M., 2008. «Qu'est-ce qu'un verbe de déplacement ? : critères spatiaux pour une classification des verbes de déplacement intransitifs du français ", in J. Durand, B. Habert (eds), Congrès Mondial de Linguistique Française - CMLF08. http :/www.linguistiquefrançaise.org (consulté 10 décembre 2009). 
Aurnague, M., 2010. « Places-repère, localisation et routines : lorsque l'analyse du nom place rejoint celle de la préposition à ", Corela, Numéros Spéciaux, Espace, préposition, cognition - hommage à Claude Vandeloise, http://www.revue-corela.org (consulté le 14 juin 2010).

Aurnague, $\mathrm{M}, 2012$. « De l'espace à l'aspect (interne) : les bases ontologiques des procès de déplacement », Corela, ce numéro, http://www.revue-corela.org.

Boons, J.-P., 1984. «Sceller un piton dans le mur ; desceller un piton du mur. Pour une syntaxe de la préfixation négative ", Langue française 62, 95-128.

Boons, J.-P., 1985. « Préliminaires à la classification des verbes locatifs : les compléments de lieu, leurs critères, leurs valeurs aspectuelles », Linguisticae Investigationes 9, 195-267.

Boons, J.-P., 1987. « La notion sémantique de déplacement dans une classification syntaxique des verbes locatifs ", Langue Française 76, 5-40.

Borillo, A., 1998. L'espace et son expression en français. Paris : Ophrys.

Crisco : Dictionnaire synonymique, www.crisco.unicaen.fr/ (consulté le 12 janvier 2010).

Gerhard-Krait, F., 2000. La préfixation en dé(s)-: Formes construites et interprétations. Thèse de doctorat. Université Marc Bloch, Strasbourg II.

Gerhard-Krait, F., 2005. «Les formations verbales préfixées par re-itératif : émergence des traits qualité et quantité », Scolia 20, 171-185.

Gerhard-Krait, F., 2010 a. « La filiation du nom d'espace place : le cas du verbe placer », Scolia 24, 21-36.

Gerhard-Krait, F., 2010 b. " Les verbes en re- itératifs ; sens et interprétation en discours ", in C.A. Alvarez, F. Bango de la Campa, M.-L. Donaire (eds), Etudes sur la combinatoire et la hiérarchie des composants. Bern, Berlin, Bruxelles, etc. : Peter Lang, 255-270.

Gerhard-Krait, F., (à paraître). " Le verbe déplacer : un dérivé atypique », Actes du $26^{\text {ème Congrès }}$ International de Linguistique et de Philologie Romanes, Valence 2010.

Geuder, W., 2009. «Descendre en grimpant : une étude contrastive de l'interaction entre déplacement et « manière de mouvement » ", Langages 175, 103-121.

Laur, D., 1991. Sémantique du déplacement et de la localisation en français : une étude des prépositions et de leur relation dans la phrase simple. Thèse de doctorat. Université de Toulouse Le Mirail.

Laur, D., 1993. «La relation entre le verbe et la préposition dans la sémantique du déplacement », Langages 110, 47-67.

Huyghe, R., 2009. Les Noms Généraux d'Espace. Bruxelles : de Boeck, Duculot.

Jalenques, P., 2000. Contribution à l'étude du préfixe RE en français contemporain : pour une analyse compositionnelle du verbe regarder. Thèse de doctorat. Université Denis Diderot, Paris 7.

Jalenques, P., 2001, «Quand la diachronie renvoie à la synchronie : études des emplois idiomatiques du préfixe re en français (renier, remarquer, regarder, etc.) », Recherches linguistiques de Vincennes 30, 39-61.

Jalenques, P., 2002. «Etude sémantique du préfixe RE en français contemporain : à propos de plusieurs débats actuels en morphologie dérivationnelles », Langue Française 133, 74-90.

Kleiber, G., 1987. Du côté de la référence verbale. Les phrases habituelles. Berne : Peter Lang. 
Kopecka, A., 2009. «L'expression du déplacement en français : l'interaction des facteurs sémantiques, aspectuels et pragmatiques dans la construction du sens spatial », Langages 173, 54-73.

Mascherin, L., 2007. Analyse morphosémantique de l'aspectuo-temporalité en français : le cas du préfixe RE-. Thèse de doctorat. Université Nancy 2.

Sablayrolles, P., 1995. Sémantique formelle de l'expression du mouvement. Toulouse. Université de Toulouse III.

Sarda, L., 2000. «L'expression d'un déplacement dans la construction transitive directe », Syntaxe \& Sémantique 2, 121-137.

Sarda, L., 1999. Contribution à l'étude de la sémantique de l'espace et du temps : analyse des verbes de déplacement transitifs directs du français. Thèse de doctorat. Université de Toulouse Le Mirail.

Stosic, D., 2009. « La notion de manière dans la sémantique de l'espace », Langages 175, 103-121.

Vandeloise, C., 1986. L'espace en français. Paris : Seuil.

Vandeloise, C., 1999. " Une place pour chaque chose et chaque chose à sa place ", in M. Plénat, M. Aurnague, A. Condamines, J.P. Maurel, C. Molinier \& C. Muller (eds), L'emprise du sens : structures linguistiques et interprétations. Mélanges offerts à Andrée Borillo. Amsterdam : Rodopi, 303-321.

Vandeloise, C., 2001. Aristote et le lexique de l'espace : rencontres entre la physique grecque et la linguistique cognitive. Stanford, CA : CSLI.

Zribi-Hertz, A., 2008. »Le médiopassif à accord riche en français : pour une approche multifactorielle ", in J. Durand et al. (eds), CMLF'08, 2645-2662. http://dx.doi.org/10.1051/ cmlf08083.

\section{NOTES}

1. Cf. pour le verbe placer Gerhard-Krait (2010a), et pour le verbe déplacer Gerhard-Krait (à par.).

2. Nous désignons par emplois concrets, les emplois pour lesquels le changement de la relation spatiale engage une cible dénotant une entité matérielle. Et nous désignons par emplois lexicalisés, les emplois tels que replacer de l'argent (à la banque) et déplacer des populations, dont l'interprétation est globale.

3. Cf. Huyghe (2009).

4. Pour une différenciation entre les propriétés aspectuelles et les propriétés locatives, voir Sarda (2000: 122-123).

5. On relève notamment: (i) la valeur des compléments autres que le cod, les compléments d'espace (sur les compléments prépositionnels à valeur spatiale (cf. Vandeloise 1986, Borillo 1998, Laur 1991, 1993, etc.), et plus généralement les compléments de manière de mouvement (cf. Geuder 2009, Stosic 2009 par ex.), (ii) l'incidence de la valeur aspectuelle des temps, (iii) l'incidence de la fréquence explicite ou implicite (cf. Kleiber 1987 et la littérature sur la pluriactionnalité), et (iv) les propriétés ontologiques des entités spatiales site et cible impliquées dans le procès (cf. notamment les travaux de Aurnague 1996, Sarda 1999, etc.).

6. Les exemples non référencés sont des exemples construits.

7. Sur le préfixe re- et les différentes positions concernant sa valeur et son fonctionnement sémantique, voir Amiot (2002), Apothéloz (2005), Gerhard-Krait (2005, 2010b), Jalenques (2000, 2001, 2002) et Mascherin (2007).

8. La manière de mouvement se caractérise par une polarité médiane et la manière de se déplacer. Le mode de réalisation du déplacement, englobe des paramètres aussi variés que la 
vitesse, la force, la forme de la trajectoire, le moyen, le milieu servant de support, le degré d'effort déployé, etc. (cf. Stosic 2009 : 111).

9. La différence entre un site du type de place et un site du type de lieu est explicitée dans la section 2.1.

10. Le caractère axiologique négatif d'un emploi, n'est pas toujours fondé sur des données totalement tangibles. Dans l'exemple (23), il découle, me semble-t-il, à la fois : (i) de la corrélation causale qui explicite une disproportion entre une petite cause et un gros effet (puisque un mot suffit à faire déplacer des spectateurs, à mettre des spectateurs dans l'obligation de céder leur place), et (ii) de la prise en compte du contexte épistémologique, en vertu duquel le comportement en question constitue un abus de pouvoir (chasser quelqu'un de sa place).

11. Un changement d'emplacement se définit généralement comme le changement continu de position d'une cible dans l'espace.

12. On ne discutera pas ici le bien-fondé de considérer que la base dérivationnelle de ces deux verbes puisse être le nom place plutôt que le verbe placer (sur la question de la catégorisation des bases des verbes en dé(s)- voir Amiot, 2008, Gerhard-Krait, 2000 et sur le verbe placer, voir Gerhard-Krait, 2010a). On précisera simplement qu'il n'est pas utile d'en passer par une base verbale pour rendre compte de la structure morpho-sémantique de ces deux verbes. En effet, il n'est pas nécessaire de présupposer qu'une entité ait été placée (qu'elle ait fait l'objet au préalable d'un procès placer) pour être déplacer ou replacer, il suffit qu'elle ait eu une place.

13. Il n'est pas exclu de trouver des scénarios pour lesquels de tels emplois sont recevables, mais ces derniers n'infirment pas l'analyse proposée, au contraire, en constituant une lecture par défaut, ils la confirment. En somme, même si le complément dénote un lieu, ce lieu est le site englobant dans lequel la cible du procès replacer doit, à proprement parler, reprendre place.

14. On le sait, la localisation d'une cible suppose nécessairement qu'elle puisse être décrite comme statique à un moment donné, ou tout au moins suffisamment statique pour qu'on puisse la repérer.

15. On relève des schémas inverses désosser un poulet, dénerver une viande où la cible est dénotée par la base du verbe et le site par le SN cod (sur le sujet, voir Gerhard-Krait 2000).

16. Le cas du verbe déménager est intéressant. En effet, dans l'usage, il signifie souvent à la fois déménager et emménager (quitter un lieu d'habitation pour un autre), notamment lorsqu'il est employé avec un complément de localisation finale (il déménage à la campagne). Il peut toutefois ne signifier qu'un départ (Pierre déménage / Pierre s'en va / Pierre quitte son appartement).

\section{RÉSUMÉS}

Dans leurs emplois spatiaux concrets les deux verbes replacer et déplacer illustrent deux modes d'exploitation différents du sens du nom d'espace place qui entre dans leur structure. Replacer est un verbe de déplacement qui utilise et respecte au plus près les spécificités sémantiques du nom en question, notamment sa vocation à la localisation substantielle. En effet, replacer $X$ désigne un procès à polarité aspectuelle / locative finale qui consiste globalement à faire changer une substance $\mathrm{X}$ de place. Pour le verbe déplacer, si le changement de localisation concerne bien des substances, il n'est en revanche nullement restreint à un changement de localisation de type place (il peut s'agir aussi bien d'un changement de lieu ou d'emplacement), pas plus qu'il n'est 
restreint à une polarité aspectuelle ou locative particulière. Ce travail examine ce qui dans la structure sémantique de ces deux verbes permet d'expliquer ces différences de comportement.

In their concrete spatial uses, the French verbs replacer "replace, put back" and déplacer "displace, move away" illustrate two different instanciations of the name place, on which they are based. Replacer is a verb of motion, which closely follows the semantic specificity of the name in question, namely its tendency to 'substantially localize'. For instance, replacer $X$ stands for a process with a final aspectual and locative polarity, which amounts to describing a change of place for the substance X. For the verb déplacer, the change of location also affects a substance, but it does not have to be a change of place in a restrictive sense, and neither is it linked to a specific aspectual or locative polarity. The aim of this study is to find an explanation for the differences in semantic behavior between these two verbs.

\section{INDEX}

Mots-clés : sémantique des mots construits, sémantique de l'espace, verbes de déplacement, indétermination aspectuelle / locative, préfixe dé- / re-

Keywords : spatial semantics, motion verbs, locative or aspectual vagueness, dé- /re- prefix

\section{AUTEUR}

FRANCINE GERHARD-KRAIT

Université de Strasbourg - EA 1339 LiLPa-Scolia 